\title{
Superior antitumor activity of trastuzumab combined with capecitabine plus oxaliplatin in a human epidermal growth factor receptor 2-positive human gastric cancer xenograft model
}

\author{
SUGURU HARADA, MIEKO YANAGISAWA, SAORI KANEKO, KEIGO YOROZU, \\ KANAME YAMAMOTO, YOICHIRO MORIYA and NAOKI HARADA \\ Product Research Department, Chugai Pharmaceutical Co., Ltd., Kamakura, Kanagawa 247-8530, Japan
}

Received April 5, 2015; Accepted June 4, 2015

DOI: $10.3892 / \mathrm{mco} .2015 .609$

\begin{abstract}
In the treatment of human epidermal growth factor receptor 2 (HER2)-positive advanced gastric or gastroesophageal junction cancer, it has been reported that the combination of trastuzumab with capecitabine plus cisplatin, or with 5-fluorouracil (5-FU) plus cisplatin, significantly increased overall survival compared with chemotherapy alone (ToGA trial). In addition, adjuvant therapy with capecitabine plus oxaliplatin (XELOX) improved the survival of patients who received curative D2 gastrectomy (CLASSIC trial). However, the efficacy of the combination of trastuzumab with XELOX for patients with HER2-positive gastric cancer remains unknown. The aim of this study, was to investigate the efficacy of the combination of trastuzumab with XELOX in a HER2-positive human gastric cancer xenograft model. Combination treatment with these three agents (trastuzumab $20 \mathrm{mg} / \mathrm{kg}$, capecitabine $359 \mathrm{mg} / \mathrm{kg}$ and oxaliplatin $10 \mathrm{mg} / \mathrm{kg}$ ), was found to exhibit a significantly stronger antitumor activity in NCI-N87 xenografts compared with either trastuzumab or XELOX alone. In this model, treatment with trastuzumab alone or trastuzumab plus oxaliplatin enhanced the expression of thymidine phosphorylase (TP), a key enzyme in the generation of 5-FU from capecitabine in tumor tissues. In in vitro experiments, trastuzumab induced TP mRNA expression in NCI-N87 cells. In addition, NCI-N87 cells co-cultured with the natural killer (NK) cell line CD16(158V)/NK-92 exhibited increased expression of TP mRNA. When NCI-N87 cells were cultured with CD16(158V)/NK-92 cells in the presence of trastuzumab, the mRNA expression of cytokines reported to have the ability to induce TP was upregulated in tumor cells. Furthermore, a medium conditioned by CD16(158V)/NK-92 cells also
\end{abstract}

Correspondence to: Dr Naoki Harada, Product Research Department, Chugai Pharmaceutical Co., Ltd., 200 Kajiwara, Kamakura, Kanagawa 247-8530, Japan

E-mail: haradanok@chugai-pharm.co.jp

Key words: trastuzumab, thymidine phosphorylase, oxaliplatin, capecitabine upregulated the expression of TP mRNA in NCI-N87 cells. These results suggest that trastuzumab promotes TP expression, either by acting directly on NCI-N87 cells, or indirectly via a mechanism that includes trastuzumab-mediated interactions between NK and NCI-N87 cells. Therefore, the combination of trastuzumab with XELOX may be a potent therapy for HER2-positive gastric cancer.

\section{Introduction}

Gastric cancer is one of the most prevalent malignancies and the second leading cause of cancer-related mortality worldwide (1). Although the mainstay of treatment is curative surgery (2), several patients develop recurrence, even following surgery. Various adjuvant therapies have been developed to prevent postoperative recurrence (3-5). However, the efficacy of these therapies is limited and there is a need for more effective treatments for gastric cancer.

Human epidermal growth factor receptor 2 (HER2) is known to be involved in the complex signaling pathways controlling cell proliferation, differentiation and apoptosis through the activation of signaling cascades (6). The overexpression of HER2 is associated with increased metastatic potential and poor clinical outcome $(7,8)$. Trastuzumab, a humanized anti-HER2 antibody, exerts its antitumor activity via several mechanisms, including blockade of constitutive HER2 signaling, antibody-dependent cell-mediated cytotoxicity (ADCC) by tumor-infiltrating FcR-expressing immune effector cells and suppression of tumor angiogenesis (9-13). Trastuzumab is widely used as a standard therapy for patients with HER2-overexpressing metastatic breast cancer (14-16). In addition to its effect in breast cancer, trastuzumab is reported to exhibit strong antitumor activity in HER2-overexpressing human gastric cancer mouse xenograft models (17). In a phase III clinical trial of trastuzumab in HER2-positive advanced and inoperable gastric cancer (ToGA trial), compared with chemotherapy alone, treatment with trastuzumab in combination with chemotherapy (capecitabine plus cisplatin) significantly prolonged the overall survival of patients with HER2-positive advanced gastric or gastroesophageal junction cancer (18). Therefore, anti-HER2 therapy with trastuzumab is highly recommended for HER2-overexpressing gastric cancer. 
Capecitabine (N4-pentyloxycarbonyl-5'-deoxy-5fluorocytidine) is an oral fluoropyrimidine that undergoes a 3-step enzymatic activation process in hepatic and tumor tissues. The final step that generates 5-fluorouracil (5-FU) occurs selectively within tumor tissues, due to the higher expression of thymidine phosphorylase (TP) in tumors compared with that in normal tissues (19-21). As regards the metabolism of 5-FU, it is deactivated enzymatically by dihydropyrimidine dehydrogenase (DPD), which is also expressed in tumor tissues. By using human tumor xenograft models, it has been demonstrated that the sensitivity to capecitabine correlates with the expression of TP and DPD, specifically the TP/DPD ratio $(22,23)$. It has also been demonstrated in clinical studies that the levels of TP and DPD in tumors correlate with the efficacy of capecitabine (24-26). Several antitumor modalities, such as cyclophosphamide, taxanes, oxaliplatin, erlotinib, or radiation, have been reported to increase the levels of TP in tumors in xenograft models and, when these modalities are used in combination with capecitabine, they exhibit a significantly higher antitumor activity compared with each agent or treatment used as monotherapy (27-31).

Oxaliplatin is an alkylating drug that forms compounds between two adjacent guanines or a guanine and an adenine residue, leading to inhibition of DNA synthesis and repair (32). Several phase II trials have demonstrated that the combination of oral capecitabine with intravenous oxaliplatin (XELOX) is an effective and well-tolerated treatment for advanced gastric cancer (33-35). In addition, the CLASSIC trial compared XELOX with surgery alone in patients who underwent D2 gastrectomy. The interim results of that study demonstrated that XELOX improved 3-year disease-free survival compared with surgery alone (36). It has also been reported that XELOX exhibited a superior antitumor activity over either single agent in human gastrointestinal cancer xenograft models (30).

Considering the aforementioned findings, we hypothesized that a combination treatment with trastuzumab and XELOX may be a potent therapy for HER2-positive gastric cancer. However, thus far there have been no preclinical or clinical studies investigating the efficacy of this combination in gastric cancer. The aim of the present study was to assess the antitumor effect of the combination of trastuzumab with XELOX and analyze its mechanism of action from the aspect of the induction of the capecitabine-activating enzyme TP.

\section{Materials and methods}

Antitumor agents. Capecitabine and trastuzumab were obtained from Chugai Pharmaceutical Co., Ltd (Tokyo, Japan). Oxaliplatin was purchased from Wako Pure Chemical Industries (Osaka, Japan). Human $\mathrm{IgG}$ (HuIgG) was purchased from MP Biomedicals, Inc. (Aurora, OH, USA).

Animals. A total of 254, 5-week-old male CAnN. Cg-Foxn1nu/CrlCrlj mice were obtained from Charles River Laboratories Japan, Inc. (Yokohama, Japan). The mice had an average body weight of $26.3 \mathrm{~g}$ on the day of treatment initiated. The health of the mice was monitored by daily observation. Chlorinated water and irradiated food (CE-2; Clea Japan, Inc., Tokyo, Japan) were provided ad libitum and the animals were kept under a controlled light/dark cycle (12 h light; $12 \mathrm{~h}$ dark).
All the mice were allowed to acclimatize and recover from shipping-related stress for at least 1 week prior to the study. All the animal experiment protocols were reviewed and approved by the Institutional Animal Care and Use Committee at Chugai Pharmaceutical Co., Ltd.

Cell lines and culture conditions. The HER2-positive human gastric cancer cell line NCI-N87 was purchased from the American Type Culture Collection (Manassas, VA, USA) and maintained in RPMI-1640 medium supplemented with $10 \%(\mathrm{v} / \mathrm{v})$ fetal bovine serum (FBS) at $37^{\circ} \mathrm{C}$ under $5 \% \mathrm{CO}_{2}$. $\mathrm{CD} 16(158 \mathrm{~V}) / \mathrm{NK}-92$ cells were constructed as previously described (37) and maintained in MEM $\alpha$ medium (Wako Pure Chemical Industries) supplemented with $12.5 \%$ FBS, $12.5 \%$ horse serum, $0.02 \mathrm{mmol} / \mathrm{l}$ folic acid, $0.1 \mathrm{mmol} / 12$-mercaptoethanol, $0.2 \mathrm{mmol} / \mathrm{l}$ inositol, $0.5 \mathrm{mg} / \mathrm{ml} \mathrm{G} 418$ and $20 \mathrm{ng} / \mathrm{ml}$ recombinant human interleukin (IL)- 2 at $37^{\circ} \mathrm{C}$ under $5 \% \mathrm{CO}_{2}$.

In vivo tumor growth inhibition studies. Each mouse was inoculated subcutaneously into the right flank with $5 \times 10^{6}$ NCI-N87 cells. The tumor volumes (V) were estimated from the equation $\mathrm{V}=\mathrm{ab}^{2} / 2$, where $\mathrm{a}$ and $\mathrm{b}$ are the tumor length and width, respectively. Several weeks after tumor inoculation and once tumors had reached a volume of $\sim 160 \mathrm{~mm}^{3}$, the mice were randomized into 7-8 mice per treatment group, and treatment with capecitabine $(359 \mathrm{mg} / \mathrm{kg})$, oxaliplatin $(10 \mathrm{mg} / \mathrm{kg})$, trastuzumab $(20 \mathrm{mg} / \mathrm{kg})$ or HuIgG $(20 \mathrm{mg} / \mathrm{kg})$ was initiated (day 1). Capecitabine was suspended in $40 \mathrm{mmol} / \mathrm{l}$ citrate buffer ( $\mathrm{pH}$ 6.0) containing 5\% gum arabic as the vehicle and was administered orally once a day for 14 days. Oxaliplatin was dissolved in 5\% glucose and administered intravenously on day 1. Trastuzumab and HuIgG were diluted with saline and administered intraperitoneally once a week for 3 weeks. The tumor volume was measured twice a week and the degree of tumor growth inhibition was evaluated on day 22. In order to determine the levels of TP and DPD in the tumor and for immunohistochemistry (IHC), the mice bearing NCI-N87 tumors were randomized into 6 mice per treatment group and treated once with oxaliplatin and once a week with trastuzumab or HuIgG. The tumors were excised on day 15.

Measurement of TP and DPD protein levels in tumor tissues. The tumor samples obtained on day 15 were immediately frozen in liquid nitrogen and stored at $-80^{\circ} \mathrm{C}$ until use. The tumor tissues were homogenized in $10 \mathrm{mmol} / \mathrm{l}$ Tris-buffer (pH 7.4) containing $15 \mathrm{mmol} / \mathrm{l} \mathrm{NaCl}$, $1.5 \mathrm{mmol} / 1 \mathrm{MgCl}_{2}$ and $50 \mu \mathrm{mol} / 1$ potassium phosphate and were then centrifuged at $10,000 \mathrm{x}$ g for $20 \mathrm{~min}$ at $4^{\circ} \mathrm{C}$. The protein concentration of the supernatant was determined by using Direct Detect Spectrometer (Merck KGaA, Darmstadt, Germany). The levels of TP and DPD were measured by ELISA with monoclonal antibodies specific to human TP and DPD, as described previously $(38,39)$.

IHC for TP in tumor tissues. The tumors were excised on day 15 and $4-\mu \mathrm{m}$ sections were prepared from paraffin-embedded formalin-fixed tissues. IHC for TP was performed by using anti-TP antibody (anti-TYMP antibody produced in rabbit; cat. no. HPA001072, Sigma-Aldrich, St. Louis, MO, USA) and peroxidase-labeled polymer-horseradish peroxidase (HRP) 
conjugated goat anti-rabbit immunoglobulins (Envision+ kit, HRP-DAB; cat. no. K4003; Dako, Tokyo, Japan).

IHC was evaluated by scoring the positive staining strength in each mouse in the HuIgG-treated control, trastuzumab, oxaliplatin and oxaliplatin plus trastuzumab groups, and the scores were as follows: 1 , weakly positive; 2 , moderately positive; 3 , markedly positive.

In vitro reverse transcription quantitative polymerase chain reaction ( $R T-q P C R)$. To determine the direct effect of trastuzumab on the expression of capecitabine-activating enzymes, NCI-N87 cells were seeded on 6 -well plates at $8 \times 10^{5}$ cells/well and treated with $100 \mu \mathrm{g} / \mathrm{ml} \mathrm{HuIgG}$ or trastuzumab for 6,24 and $48 \mathrm{~h}(\mathrm{n}=3)$.

In the co-culture study, NCI-N87 cells were seeded on 6 -well plates at $8 \times 10^{5}$ cells/well. Following adhesion, $8 \times 10^{5} \mathrm{CD} 16(158 \mathrm{~V}) / \mathrm{NK}-92$ cells/well were transferred to the NCI-N87 plates and treated with $2 \mathrm{ng} / \mathrm{ml} \mathrm{HuIgG}$ or trastuzumab in NCI-N87 medium [CD16(158V)/NK-92 medium without G418 (1:1)] for 6 and $24 \mathrm{~h}(\mathrm{n}=3)$. Prior to extracting total RNA from NCI-N87 cells, the CD16(158V)/NK-92 cells were removed by washing with phosphate-buffered saline.

To evaluate the effects of soluble factors from CD16(158V)/NK-92 cells, these cells were seeded on 6-well plates at $8 \times 10^{5}$ cells/well and treated with HuIgG or trastuzumab $(2 \mathrm{ng} / \mathrm{ml})$ in CD16(158V)/NK-92 cell medium without G418. After $24 \mathrm{~h}$, the culture medium was collected, centrifuged and filtered. NCI-N87 cells were seeded on 6-well plates at $8 \times 10^{5}$ cells/well. Following adhesion, the NCI-N87 cells were cultured in the filtered CD16(158V)/NK-92 cell culture medium [new NCI-N87 medium (1:1)] for $24 \mathrm{~h}(\mathrm{n}=3)$.

After culturing, total RNA from the cells was extracted by using an AS2000 Maxwell 16 Instrument (Promega, Madison, WI, USA). The cDNA was synthesized from total RNA by a Transcriptor First Strand cDNA Synthesis kit (Roche Diagnostics, Indianapolis, IN, USA). The expression of TP was detected by using SYBR-Green (Roche Diagnostics). The expression of GAPDH, interferon (IFN)- $\gamma$, tumor necrosis factor (TNF)- $\alpha$ and IL-1 $\alpha$ was detected by using the Universal Probe Library (Roche Diagnostics). GAPDH was used as control.

Statistical analysis. Data are presented as the mean \pm standard deviation. For in vivo studies with three or four groups, statistical differences between individual groups were evaluated with Steel-Dwass tests. For in vivo studies with two groups, statistical comparisons between the control and trastuzumab groups were performed by t-tests. In in vitro studies, the mRNA levels of the control or treatment groups were compared to that at $0 \mathrm{~h}$ by using the Dunnett's test. The mRNA levels of the treatment groups were compared to that of the control group at the same time point using t-tests. For all the tests, $\mathrm{P}<0.05$ was considered to indicate statistically significant differences. The statistical analysis was performed using a SAS preclinical package (SAS Institute, Cary, NC, USA).

\section{Results}

Combination of trastuzumab with XELOX. The antitumor activity of trastuzumab $(20 \mathrm{mg} / \mathrm{kg})$ in combination with capecitabine $[359 \mathrm{mg} / \mathrm{kg}, 2 / 3$ maximum tolerated dose
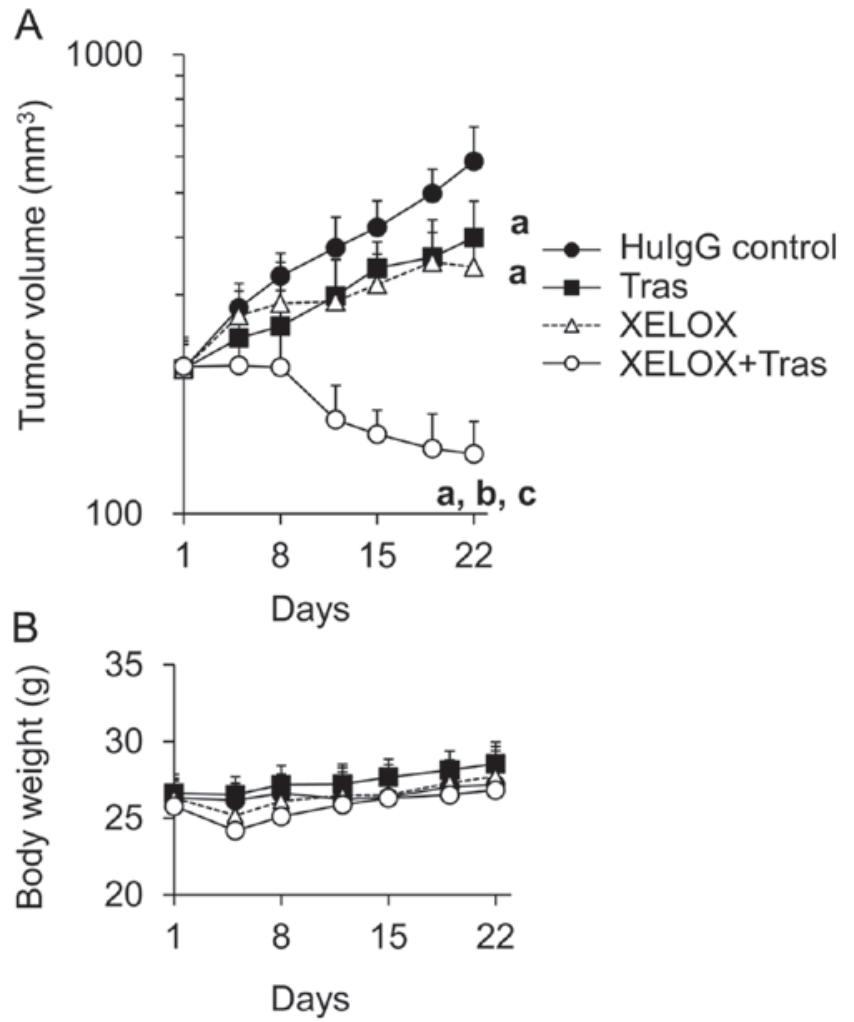

Figure 1. Effect of trastuzumab in combination with capecitabine and oxaliplatin on tumor growth in NCI-N87 xenograft model. (A) Antitumor activity in terms of tumor volume reduction and (B) treatment toxicity in terms of body weight reduction with the combination of trastuzumab and XELOX in a xenograft model of human HER2-positive gastric cancer. The values represent the mean \pm standard deviation $(n=7-8$ per treatment group). ${ }^{\text {a }} \mathrm{P}<0.05$ vs. HuIgG-treated control; ${ }^{\mathrm{b}} \mathrm{P}<0.05$ vs. trastuzumab; and ${ }^{\mathrm{c}} \mathrm{P}<0.05$ vs. XELOX (Steel-Dwass test). HuIgG, human immunoglobulin G; Tras, trastuzumab; XELOX, capecitabine plus oxaliplatin.

(MTD)] (22) and oxaliplatin (10 mg/kg, 2/3 MTD) (30) was evaluated in a HER2-positive human gastric cancer NCI-N87 xenograft model. The tumors in all the treatment groups were significantly smaller compared with those in the HuIgG-treated control group on day 22. Combined treatment with trastuzumab and XELOX achieved a significantly stronger inhibition of tumor growth compared with either trastuzumab or XELOX alone on day 22 (Fig. 1A). In addition, no augmentation of toxicity, as shown by body weight loss, was observed in any of the treatment groups (Fig. 1B).

Effect of trastuzumab on TP expression in tumor tissues. ELISA was used to investigate the effects of trastuzumab on the TP/DPD protein ratio in whole tumor tissues sampled from the NCI-N87 xenograft models on day 15. However, there was no significant difference in the TP/DPD ratio between tumor tissues obtained from mice treated with trastuzumab and those treated with HuIgG alone (Fig. 2A). As whole tumor tissues are composed of vital as well as necrotic areas, measuring TP/DPD using whole tumor tissues may not return accurate results. In order to evaluate the TP/DPD ratio more accurately, we performed an IHC examination of TP and measured the TP expression level in the vital tumor cell area. The number of TP-positive cells (brown-tinged cells) in tumors from trastuzumab-treated mice were increased compared 
A

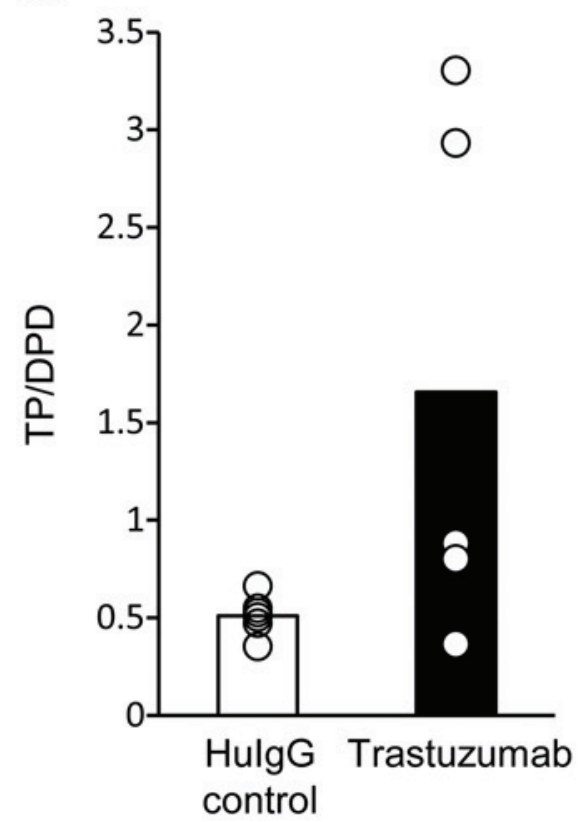

B

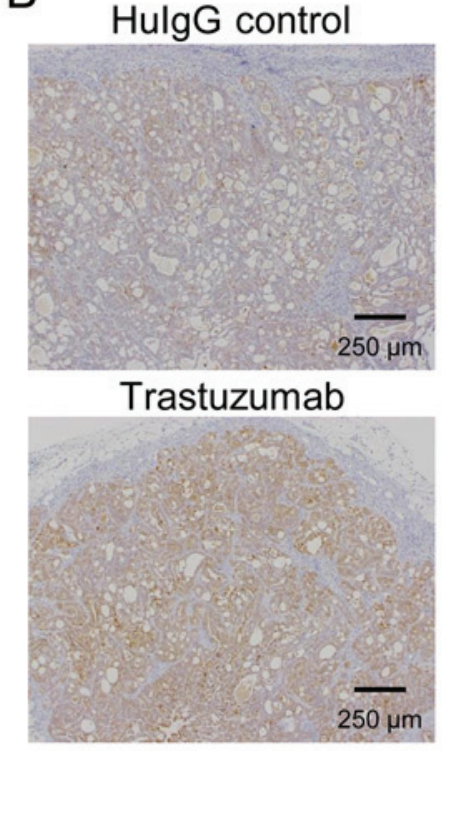

C

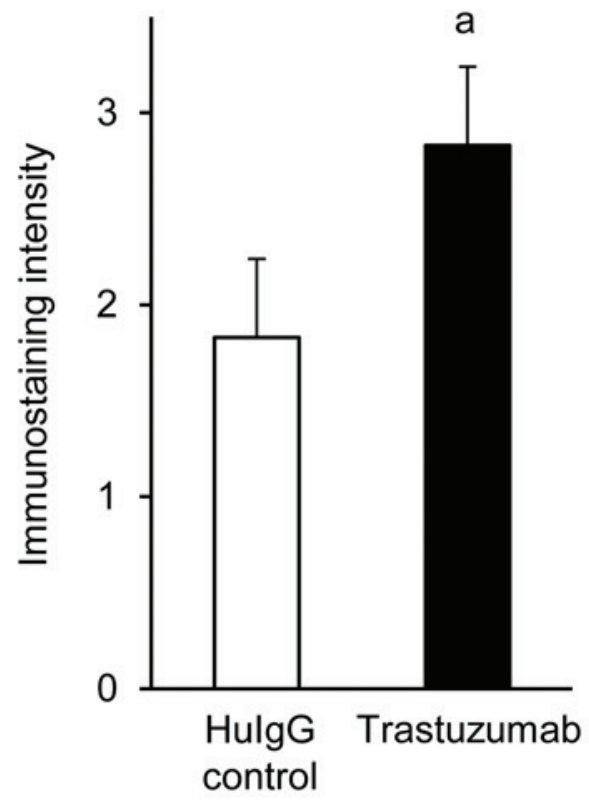

Figure 2. Upregulation of TP expression by trastuzumab in tumors of human HER2-positive gastric cancer xenografts. (A) Mean TP/DPD value in tumors treated with trastuzumab or HuIgG. The open circles represent individual data (n=6/group); (B) anti-TP antibody-stained tumor tissues (TP/IHC) from xenograft models treated with trastuzumab or HuIgG; (C) quantified immunostaining intensity of TP/IHC. The values represent the mean \pm standard deviation ( $\mathrm{n}=6$ /group); ${ }^{a} \mathrm{P}<0.05$ vs. HuIgG-treated control by Wilcoxon test. TP, thymidine phosphorylase; DPD, dihydropyrimidine dehydrogenase; HuIgG, human immunoglobulin G; IHC, immunohistochemistry.

with the respective number in tumors from HuIgG-treated mice (Fig. 2B). The score of positive staining strength was significantly higher in the trastuzumab group compared with that in the HuIgG-treated control group (Fig. 2C).

We next used ELISA to investigate the effects of oxaliplatin monotherapy and oxaliplatin in combination with trastuzumab on the TP/DPD ratio in whole tumor tissues obtained from the NCI-N87 models on day 15 . The TP/DPD ratio was increased in the oxaliplatin monotherapy group and in the oxaliplatin plus trastuzumab combination group, as compared with the ratio in the HuIgG-treated control group, although the difference was not significant (Fig. 3A). However, in IHC, the TP immunostaining intensity was very strong in tumors obtained from mice treated with the combination of trastuzumab plus oxaliplatin, whereas the staining intensity of tumor tissues from oxaliplatin-treated or HuIgG-treated mice was weaker and of a similar level (Fig. 3B). The score of positive staining strength for TP was significantly higher in the combination group compared with that in the HuIgG-treated control or oxaliplatin groups. There was no significant difference in the score between the HuIgG-treated control and oxaliplatin groups (Fig. 3C).

Effects of trastuzumab on TP mRNA expression. We first examined the effects of trastuzumab treatment on the expression of TP by using qPCR. In a 48-h culture, the TP mRNA expression level in NCI-N87 cells was significantly increased compared with the initial level, irrespective of the presence of trastuzumab $(100 \mu \mathrm{g} / \mathrm{ml})$. However, trastuzumab treatment significantly increased the TP mRNA expression compared with that without trastuzumab (Fig. 4A).

Second, in the in vitro co-culture study of NCI-N87 and CD16(158V)/NK-92 cells, the TP mRNA expression
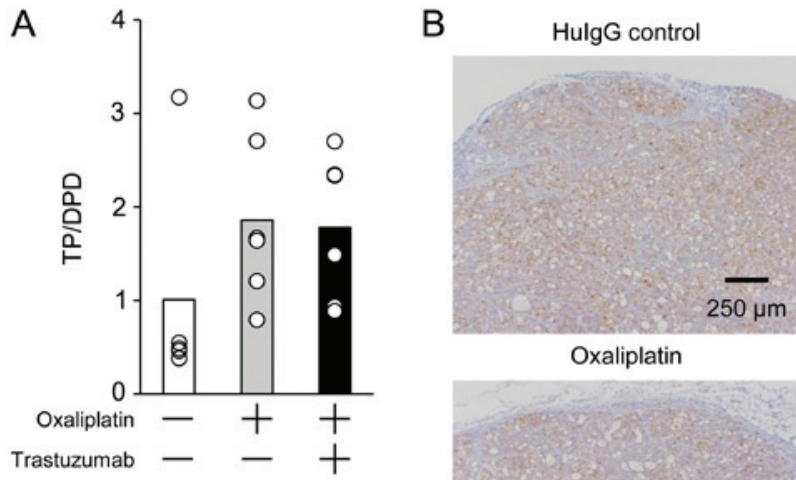

Oxaliplatin
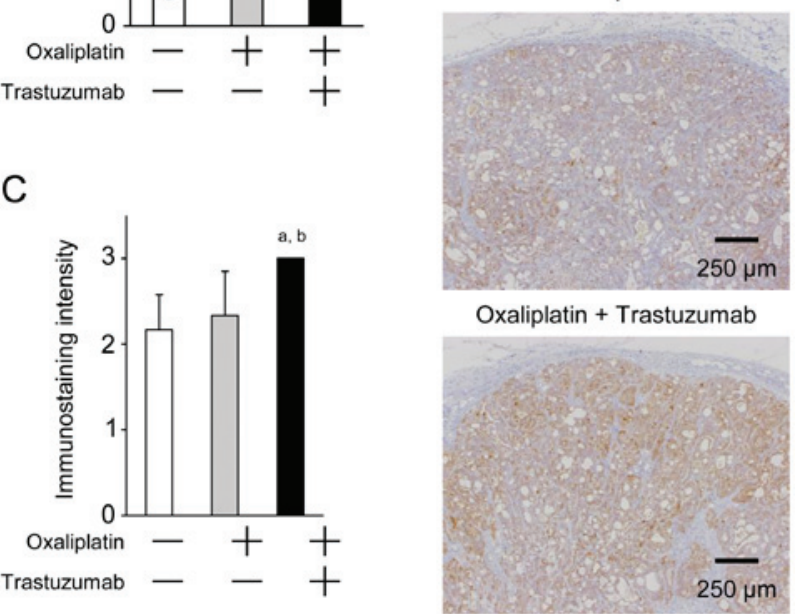

Figure 3. Upregulation of TP expression by trastuzumab plus oxaliplatin in tumors of human HER2-positive gastric cancer xenografts. (A) Mean TP/DPD ratio in tumors from mice treated with $\mathrm{HuIgG}$, oxaliplatin, or oxaliplatin plus trastuzumab. The open circles represent individual data; (B) TP/IHC of tumor tissues obtained from xenograft models treated with $\mathrm{HuIgG}$, oxaliplatin, or oxaliplatin plus trastuzumab; (C) quantified immunostaining intensity of TP/IHC. The values represent mean \pm standard deviation ( $n=6 /$ group); ${ }^{\mathrm{a}} \mathrm{P}<0.05$ vs. HuIgG-treated control; and ${ }^{\mathrm{b}} \mathrm{P}<0.05$ vs. oxaliplatin (Steel-Dwass test). TP, thymidine phosphorylase; DPD, dihydropyrimidine dehydrogenase; HuIgG, human immunoglobulin G; IHC, immunohistochemistry. 
A
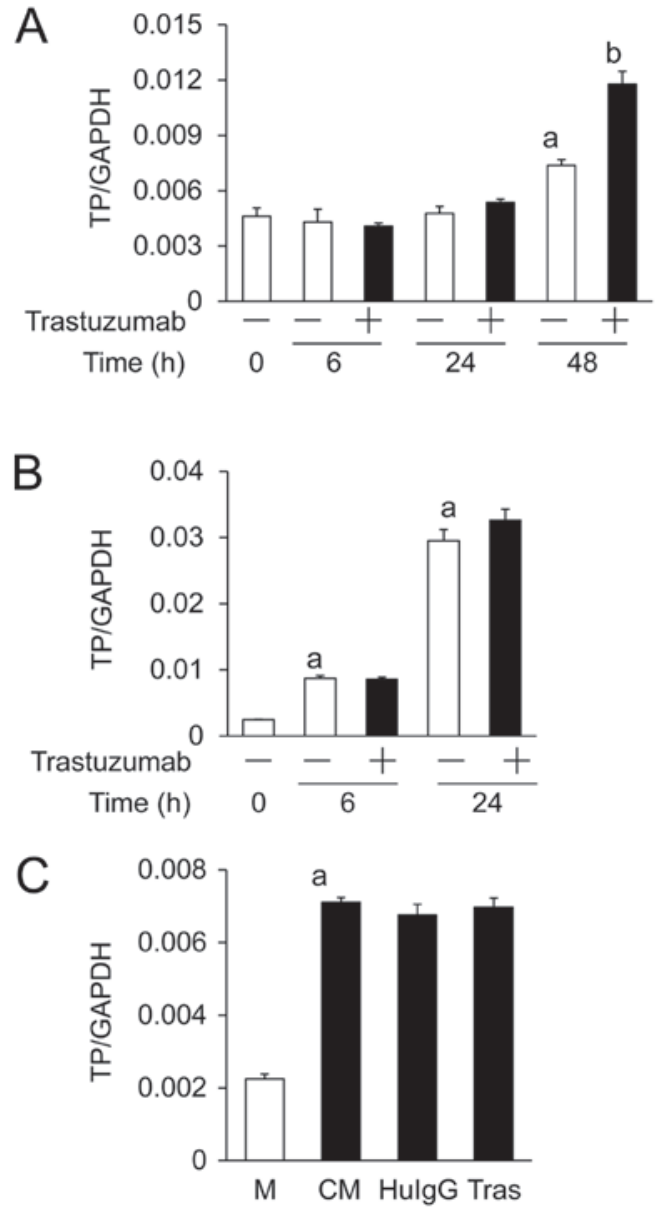

Figure 4. Effects of trastuzumab on the expression of capecitabine-activating enzymes in in vitro culture. (A) NCI-N87 cells were treated with $100 \mu \mathrm{g} / \mathrm{ml} \mathrm{HuIgG}$ (control) or trastuzumab for 6,24 and $48 \mathrm{~h}$. Total RNA was prepared from the cells and subjected to quantitative polymerase chain reaction (qPCR) analysis for TP. The values represent the mean \pm standard deviation (SD) ( $\mathrm{n}=3$ for each treatment group); ${ }^{\mathrm{a}} \mathrm{P}<0.05$ vs. $0 \mathrm{~h}$ control by Dunnett's test; and ${ }^{b} \mathrm{P}<0.05$ vs. $48 \mathrm{~h}$ control by t-test. (B) NCI-N87 and CD16(158V)/NK-92 cells were treated with $2 \mathrm{ng} / \mathrm{ml} \mathrm{HuIgG} \mathrm{(control)} \mathrm{or}$ trastuzumab for 6 and $24 \mathrm{~h}$. Total RNA was prepared from the NCI-N87 cells and subjected to qPCR for TP. The values represent the mean $\pm \mathrm{SD}(\mathrm{n}=3$ for each treatment group); ${ }^{\mathrm{P}}<0.05$ vs. $0 \mathrm{~h}$ control by Dunnett's test. (C) NCI-N87 cells were treated for $24 \mathrm{~h}$ with medium alone (M), medium conditioned by CD16(15 V)/NK-92 cells alone (CM), conditioned medium prepared in the

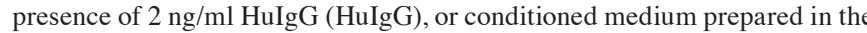
presence of trastuzumab $(2 \mathrm{ng} / \mathrm{ml})$ (Tras). Total RNA was prepared from the NCI-N87 cells and subjected to qPCR for TP. The values represent the mean $\pm \mathrm{SD}\left(\mathrm{n}=3\right.$ for each treatment group); ${ }^{\mathrm{a}} \mathrm{P}<0.05$ vs. $\mathrm{M}$ by $\mathrm{t}$-test. $\mathrm{TP}$, thymidine phosphorylase; HuIgG, human immunoglobulin G.

in NCI-N87 cells was significantly higher at 6 and $24 \mathrm{~h}$ compared with that at $0 \mathrm{~h}$, regardless of the presence of trastuzumab $(2 \mathrm{ng} / \mathrm{ml})$. The addition of trastuzumab did not affect the TP mRNA expression at either $6 \mathrm{~h}$ or $24 \mathrm{~h}$ (Fig. 4B).

Third, we used the co-culture system to investigate the effects of trastuzumab on the mRNA expression of three cytokines known to induce TP in tumor cells; the mRNA expressions of IFN- $\gamma$, TNF- $\alpha$ and IL- $1 \alpha$ at $6 \mathrm{~h}$ were significantly higher in trastuzumab-treated NCI-N87 cells compared with the HuIgG-treated cells (Fig. 5).

Finally, we investigated the effects of soluble factors from CD16(158V)/NK-92 cells on TP mRNA expression in NCI-N87 cells. Treatment for $24 \mathrm{~h}$ with a medium conditioned

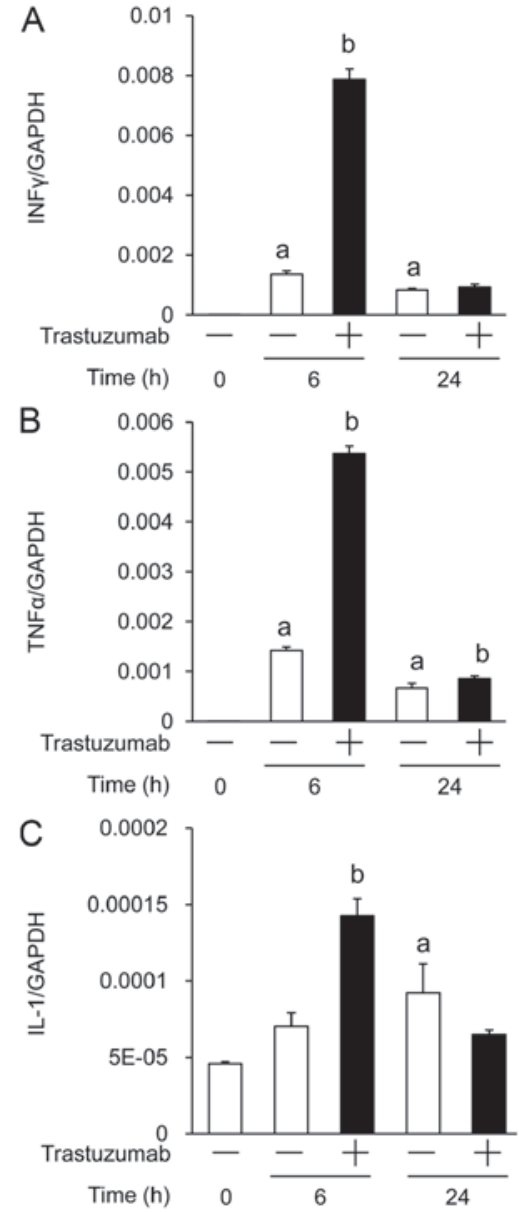

Figure 5. Effects of trastuzumab on the expression of cytokines with known ability to upregulate thymidine phosphorylase (TP), in the co-culture of NCI-N87 and CD16(158V)/NK-92 cells. NCI-N87 and CD16(158V)/NK-92 cells were treated with $2 \mathrm{ng} / \mathrm{ml} \mathrm{HuIgG} \mathrm{(control)} \mathrm{or}$ trastuzumab for 6 or $24 \mathrm{~h}$. Total RNA was prepared from NCI-N87 cells and subjected to a quantitative polymerase chain reaction analysis for (A) INF- $\gamma$, (B) TNF- $\alpha$ and (C) IL-1 $\alpha$. The values represent the mean \pm SD ( $n=3$ for each treatment group). ${ }^{a} \mathrm{P}<0.05$ vs. $0 \mathrm{~h}$ control by Dunnett's test; and ${ }^{b} \mathrm{P}<0.05$ vs. HuIgG control by t-test. HuIgG, human immunoglobulin G; IL, interleukin; INF, interferon; TNF, tumor necrosis factor.

by CD16(158V)/NK-92 cells significantly increased TP mRNA expression in NCI-N87 cells. There was no significant difference in TP mRNA expression between conditioned media prepared with trastuzumab, HuIgG, or without antibodies (Fig. 4C).

\section{Discussion}

In the present study, using the HER2-positive human gastric cancer NCI-N87 xenograft model, we investigated the efficacy of the combination of trastuzumab with XELOX and analyzed the mechanism underlying the effects of this combination in terms of capecitabine activation. We observed that, in this model, combination treatment with trastuzumab and XELOX exerted a significantly stronger antitumor effect compared with either agent alone. This effect was considered to be synergistic. In the trastuzumab group, there was an obvious antitumor effect similar to that previously reported (17). However, XELOX exerted a weaker antitumor effect compared with that previously reported in a study using human colon cancer and HER2-negative gastric cancer xenograft models (30). This 
discrepancy between the results of that study and ours may be due to the different XELOX administration schedules and the different types of cancer.

To elucidate the mechanism underlying the potent antitumor effect of the combination therapy with trastuzumab and XELOX in our model, we conducted experiments focusing on factors that facilitate the generation of 5-FU from capecitabine. For this purpose, we measured TP and DPD levels in the tumors.

In the present study, the results of IHC, which was performed with the utmost care, revealed that the administration of trastuzumab alone or trastuzumab plus oxaliplatin significantly increased the TP levels in the tumor tissues. However, no significant difference in the TP/DPD protein ratio was observed between the HuIgG-treated control and the trastuzumab groups (Fig. 2A), or between the HuIgG-treated control, oxaliplatin and trastuzumab plus oxaliplatin groups (Fig. 3A). Similar results regarding this mismatch between the results obtained by IHC and those obtained by ELISA have been previously reported (30). The discrepancy between the two methods may be explained as follows: In ELISA, the TP protein expression is represented as a value relative to the total protein in whole tumor tissues. Accordingly, the samples used for ELISA may contain proteins from connective tissue and necrotic areas, along with the vital tumor cells. On the other hand, in the IHC assay, evaluation of TP is performed by means of immunostaining intensity specifically focusing on the vital tumor cell area in the tumor tissues. Thus, we consider the results of IHC to be more reliable compared with those of ELISA for explaining the antitumor mechanism of the combination therapy. Therefore, the superior antitumor activity of the trastuzumab plus XELOX combination may be, at least in part, attributable to the increased TP levels in tumors induced by trastuzumab or trastuzumab plus oxaliplatin, which consequently facilitated the generation of 5-FU from capecitabine.

The mechanism through which TP is upregulated by trastuzumab or trastuzumab plus oxaliplatin is of great interest. We considered two possible mechanisms through which TP may be upregulated by trastuzumab: Trastuzumab acting directly on tumor cells to upregulate the expression of TP; or trastuzumab acting on other cells to release soluble factors, thereby indirectly upregulating TP expression in tumor cells. We performed several in vitro experiments to analyze these mechanisms and obtained results that may support either or both mechanisms.

When NCI-N87 cells were cultured in the presence of a relatively high concentration of trastuzumab $(100 \mu \mathrm{g} / \mathrm{ml})$, upregulation of TP mRNA in NCI-N87 cells was observed after $48 \mathrm{~h}$ of culture compared with TP mRNA in NCI-N87 cells cultured without trastuzumab, suggesting that trastuzumab acts directly to upregulate TP in HER2-positive NCI-N87 cells in vitro (Fig. 4A).

To analyze indirect upregulation of TP by trastuzumab, we conducted the following experiments focusing on tumor-infiltrating NK cells, as it is considered that ADCC is one of the key mechanisms underlying the antitumor activity of trastuzumab (40). Studies using the human NK cell line NK-92 and the CD16-transfected human NK cell line CD16(158V)/NK-92 as effector cells have indicated that trastuzumab triggers ADCC against HER2-positive human breast cancer and human gastric cancer cell lines $(37,41)$; in addition, in clinical studies, increased numbers of tumor-infiltrating NK cells have been detected in breast cancer tissues following trastuzumab treatment $(42,43)$. In light of these reports, we performed a co-culture experiment to determine whether NK cells affect TP expression in tumor cells. Specifically, NCI-N87 and CD16(158V)/NK-92 cells were co-cultured in the presence or absence of $2 \mathrm{ng} / \mathrm{ml}$ trastuzumab. The concentration of trastuzumab used in our study was the concentration used in the ADCC assay reported previously (37). It was observed that co-culture significantly increased the expression of TP mRNA in NCI-N87 cells by as early as $6 \mathrm{~h}$ of culture, and more strongly increased TP mRNA expression at $24 \mathrm{~h}$. However, the effect that co-culture without trastuzumab exerted on increasing TP mRNA expression was almost identical to that of co-culture with trastuzumab (Fig. 4B). This may be due to the strong TP-inducing effect of CD16(158V)/NK-92 cells alone, or due to the low trastuzumab concentration used in this assay. The results suggested that CD16(158V)/NK-92 cells spontaneously produce soluble factors that may act on NCI-N87 cells to upregulate TP. To investigate this hypothesis, we next examined the TP-inducing activity of CD16(158V)/NK-92-conditioned medium. As expected, TP mRNA expression in NCI-N87 cells was significantly increased by culturing in a medium conditioned by CD16(158V)/NK-92 cells (Fig. 4C). Interestingly, the induction of TP mRNA in NCI-N87 cells was similar in the conditioned medium prepared in the presence as well as in the absence of trastuzumab. These results suggested that soluble factors responsible for inducing TP mRNA expression in NCI-N87 cells were constitutively produced by CD16(158V)/NK-92 cells and their production was not affected by trastuzumab.

As regards the soluble factors that induce TP, it has been reported that the expression of TP in tumor cells is induced by treatment with cytokines, such as IL-1 $\alpha$, TNF- $\alpha$ and IFN- $\gamma$ (44). Therefore, we measured the mRNA levels of these cytokines in NCI-N87 cells co-cultured with CD16(158V)/NK-92 cells (Fig. 5). Of note, when co-culture was performed with trastuzumab for $6 \mathrm{~h}$, the mRNA levels of IFN- $\gamma, \mathrm{TNF} \alpha$ and IL-1 were significantly increased compared with those in the co-culture without trastuzumab $(\mathrm{P}<0.05)$. In the 24-h co-culture, the mRNA level of each of the cytokines decreased from that in the 6-h co-culture (Fig. 5). There appears to be some inconsistency between these results and the induction of TP in the co-culture experiment (Fig. 4B) in two respects: The first is the lag between cytokine production and TP induction; the second is the inconsistency in the effects of trastuzumab, i.e., although the levels of production of the three cytokines were higher in the presence of trastuzumab, the TP mRNA expression level was almost identical in the presence as well as in the absence of trastuzumab. With respect to the first issue, it may be considered that there is a delay for NCI-N87 cells to produce TP in response to the cytokines produced after $\sim 6 \mathrm{~h}$ of co-culture. Albanell et al (45) reported that IFN- $\gamma$, TNF- $\alpha$, or IL-1 upregulated TP when the WiDr and MKN45 human gastric cancer cell lines were cultured for $1 \mathrm{~h}$ with these cytokines, either as single agents or in combination. The second issue may be interpreted as a result of the co-culture period; the difference in TP mRNA expression in NCI-N87 cells co-cultured with or without trastuzumab may become clearer when the culture period is shorter or longer than $24 \mathrm{~h}$. 
Taken together, these data suggest that the tumor-infiltrating NK cells play an important role in the expression of TP.

In this study, we clearly demonstrated that the antitumor activity achieved with the combination of trastuzumab and XELOX was significantly greater compared with that with trastuzumab or XELOX alone in this HER2-positive human gastric cancer xenograft model. This synergistic effect was considered to be attributable, at least in part, to the upregulation of TP levels in tumors caused by trastuzumab or trastuzumab plus oxaliplatin, as the upregulation of TP facilitates the conversion of capecitabine into 5-FU in tumors. In addition, our study revealed two mechanisms through which trastuzumab upregulated TP in tumor cells: One was a direct mechanism, in which trastuzumab binding to HER2-positive tumor cells directly upregulated TP in the target tumor cells; the other was an indirect mechanism, in which trastuzumab-mediated release of soluble factors from tumor-infiltrating immune cells promoted the upregulation of TP in the HER2-positive gastric tumor cells. Thus far, it remains unclear which of these two is the principal mechanism and which is the pathway to TP upregulation. Although these issues remain to be addressed, it is expected that trastuzumab will be clinically used in combination with XELOX for HER2-positive gastric cancer in the foreseeable future.

\section{References}

1. Hartgrink HH, Jansen EP, van Grieken NC and van de Velde CJ: Gastric cancer. Lancet 374: 477-490, 2009.

2. Sasako M, Saka M, Fukagawa T, Katai H and Sano T: Surgical treatment of advanced gastric cancer: Japanese perspective. Dig Surg 24: 101-107, 2007.

3. Cunningham D, Allum WH, Stenning SP, et al; MAGIC Trial Participants: Perioperative chemotherapy versus surgery alone for resectable gastroesophageal cancer. N Engl J Med 355: 11-20, 2006.

4. Cunningham D, Starling N, Rao S, et al; Upper Gastrointestinal Clinical Studies Group of the National Cancer Research Institute of the United Kingdom: Capecitabine and oxaliplatin for advanced esophagogastric cancer. N Engl J Med 358: 36-46, 2008.

5. Macdonald JS, Smalley SR, Benedetti J, et al: Chemoradiotherapy after surgery compared with surgery alone for adenocarcinoma of the stomach or gastroesophageal junction. N Engl J Med 345 725-730, 2001

6. Yarden Y and Sliwkowski MX: Untangling the ErbB signalling network. Nat Rev Mol Cell Biol 2: 127-137, 2001.

7. García I, Vizoso F, Martín A, et al: Clinical significance of the epidermal growth factor receptor and HER2 receptor in resectable gastric cancer. Ann Surg Oncol 10: 234-241, 2003

8. Tanner M, Hollmen M, Junttila TT, et al: Amplification of HER 2 in gastric carcinoma: association with topoisomerase IIalpha gene amplification, intestinal type, poor prognosis and sensitivity to trastuzumab. Ann Oncol 16: 273-278, 2005.

9. Molina MA, Codony-Servat J, Albanell J, et al: Trastuzumab (herceptin), a humanized anti-Her2 receptor monoclonal antibody, inhibits basal and activated Her 2 ectodomain cleavage in breast cancer cells. Cancer Res 61: 4744-4749, 2001.

10. Izumi $\mathrm{Y}, \mathrm{Xu} \mathrm{L}$, di Tomaso E, Fukumura D and Jain RK: Tumour biology: Herceptin acts as an anti-angiogenic cocktail. Nature 416: 279-280, 2002.

11. Barok M, Isola J, Pályi-Krekk Z, et al: Trastuzumab causes antibody-dependent cellular cytotoxicity-mediated growth inhibition of submacroscopic JIMT-1 breast cancer xenografts despite intrinsic drug resistance. Mol Cancer Ther 6: 2065-2072, 2007.

12. Valabrega G, Montemurro F and Aglietta M: Trastuzumab: mechanism of action, resistance and future perspectives in HER2-overexpressing breast cancer. Ann Oncol 18: 977-984, 2007.

13. Musolino A, Naldi N, Bortesi B, et al: Immunoglobulin G fragment $\mathrm{C}$ receptor polymorphisms and clinical efficacy of trastuzumab-based therapy in patients with HER-2/neu-positive metastatic breast cancer. J Clin Oncol 26: 1789-1796, 2008.
14. Buzdar AU, Valero V, Ibrahim NK, et al: Neoadjuvant therapy with paclitaxel followed by 5 -fluorouracil, epirubicin and cyclophosphamide chemotherapy and concurrent trastuzumab in human epidermal growth factor receptor 2-positive operable breast cancer: An update of the initial randomized study population and data of additional patients treated with the same regimen. Clin Cancer Res 13: 228-233, 2007.

15. Romond EH, Perez EA, Bryant J, et al: Trastuzumab plus adjuvant chemotherapy for operable HER2-positive breast cancer. N Engl J Med 353: 1673-1684, 2005.

16. Smith I, Procter M, Gelber RD, et al; HERA study team: Two year follow-up of trastuzumab after adjuvant chemotherapy in HER2-positive breast cancer: A randomised controlled trial. Lancet 369: 29-36, 2007.

17. Fujimoto-Ouchi K, Sekiguchi F, Yasuno H, Moriya Y, Mori K and Tanaka Y: Antitumor activity of trastuzumab in combination with chemotherapy in human gastric cancer xenograft models. Cancer Chemother Pharmacol 59: 795-805, 2007.

18. Bang YJ, Van Cutsem E, Feyereislova A, et al; ToGA Trial Investigators: Trastuzumab in combination with chemotherapy versus chemotherapy alone for treatment of HER2-positive advanced gastric or gastro-oesophageal junction cancer (ToGA): A phase 3, open-label, randomised controlled trial. Lancet 376: 687-697, 2010.

19. Ishikawa T, Utoh M, Sawada N, et al: Tumor selective delivery of 5-fluorouracil by capecitabine, a new oral fluoropyrimidine carbamate, in human cancer xenografts. Biochem Pharmacol 55: 1091-1097, 1998

20. Pentheroudakis $\mathrm{G}$ and Twelves $\mathrm{C}$ : The rational development of capecitabine from the laboratory to the clinic. Anticancer Res 22: 3589-3596, 2002.

21. Schüller J, Cassidy J, Dumont E, et al: Preferential activation of capecitabine in tumor following oral administration to colorectal cancer patients. Cancer Chemother Pharmaco 45: 291-297, 2000.

22. Ishikawa T, Sekiguchi F, Fukase $\mathrm{Y}$, Sawada N and Ishitsuka H: Positive correlation between the efficacy of capecitabine and doxifluridine and the ratio of thymidine phosphorylase to dihydropyrimidine dehydrogenase activities in tumors in human cancer xenografts. Cancer Res 58: 685-690, 1998.

23. Yasuno H, Kurasawa M, Yanagisawa M, Sato Y, Harada N and Mori K: Predictive markers of capecitabine sensitivity identified from the expression profile of pyrimidine nucleoside-metabolizing enzymes. Oncol Rep 29: 451-458, 2013.

24. Ishii R, Takiguchi N, Oda K, Koda K and Miyazaki M: Thymidine phosphorylase expression is useful in selecting adjuvant chemotherapy for stage III gastric cancer. Int J Oncol 19: 717-722, 2001.

25. Nishimura G, Terada I, Kobayashi T, et al: Thymidine phosphorylase and dihydropyrimidine dehydrogenase levels in primary colorectal cancer show a relationship to clinical effects of 5'-deoxy-5-fluorouridine as adjuvant chemotherapy. Oncol Rep 9: 479-482, 2002.

26. Terashima M, Fujiwara H, Takagane A, et al: Role of thymidine phosphorylase and dihydropyrimidine dehydrogenase in tumour progression and sensitivity to doxifluridine in gastric cancer patients. Eur J Cancer 38: 2375-2381, 2002.

27. Endo M, Shinbori N, Fukase Y, et al: Induction of thymidine phosphorylase expression and enhancement of efficacy of capecitabine or 5'-deoxy-5-fluorouridine by cyclophosphamide in mammary tumor models. Int J Cancer 83: 127-134, 1999.

28. Sawada N, Ishikawa T, Fukase Y, Nishida M, Yoshikubo T and Ishitsuka $\mathrm{H}$ : Induction of thymidine phosphorylase activity and enhancement of capecitabine efficacy by taxol/taxotere in human cancer xenografts. Clin Cancer Res 4: 1013-1019, 1998.

29. Sawada N, Ishikawa T, Sekiguchi F, Tanaka Y and Ishitsuka H: X-ray irradiation induces thymidine phosphorylase and enhances the efficacy of capecitabine (Xeloda) in human cancer xenografts. Clin Cancer Res 5: 2948-2953, 1999.

30. Sawada N, Kondoh K and Mori K: Enhancement of capecitabine efficacy by oxaliplatin in human colorectal and gastric cancer xenografts. Oncol Rep 18: 775-778, 2007.

31. Ouchi KF, Yanagisawa M, Sekiguchi F and Tanaka Y: Antitumor activity of erlotinib in combination with capecitabine in human tumor xenograft models. Cancer Chemother Pharmacol 57: 693-702, 2006.

32. Woynarowski JM, Faivre S, Herzig MC, et al: Oxaliplatin-induced damage of cellular DNA. Mol Pharmacol 58: 920-927, 2000.

33. Dong N, Jiang W, Li H, Liu Z, Xu X and Wang M: Triweekly oxaliplatin plus oral capecitabine as first-line chemotherapy in elderly patients with advanced gastric cancer. Am J Clin Oncol 32: 559-563, 2009 
34. Liu C, Sun Q, Hang X, Zhong B and Wang D: Multicenter phase II study of capecitabine plus oxaliplatin as a first-line therapy in Chinese patients with advanced gastric cancer. Anticancer Drugs 19: 825-831, 2008.

35. Park YH, Lee JL, Ryoo BY, et al: Capecitabine in combination with oxaliplatin (XELOX) as a first-line therapy for advanced gastric cancer. Cancer Chemother Pharmacol 61: 623-629, 2008.

36. Bang YJ, Kim YW, Yang HK, et al; CLASSIC trial investigators: Adjuvant capecitabine and oxaliplatin for gastric cancer after D2 gastrectomy (CLASSIC): A phase 3 open-label, randomised controlled trial. Lancet 379: 315-321, 2012.

37. Yamashita Kashima Y, Iijima S, Yorozu K, et al: Pertuzumab in combination with trastuzumab shows significantly enhanced antitumor activity in HER2-positive human gastric cancer xenograft models. Clin Cancer Res 17: 5060-5070, 2011.

38. Mori K, Hasegawa M, Nishida M, et al: Expression levels of thymidine phosphorylase and dihydropyrimidine dehydrogenase in various human tumor tissues. Int J Oncol 17: 33-38, 2000.

39. Nishida M, Hino A, Mori K, Matsumoto T, Yoshikubo T and Ishitsuka H: Preparation of anti-human thymidine phosphorylase monoclonal antibodies useful for detecting the enzyme levels in tumor tissues. Biol Pharm Bull 19: 1407-1411, 1996.

40. Spector NL and Blackwell KL: Understanding the mechanisms behind trastuzumab therapy for human epidermal growth factor receptor 2-positive breast cancer. J Clin Oncol 27: 5838-5847, 2009.
41. Kute TE, Savage L, Stehle JR Jr, et al: Breast tumor cells isolated from in vitro resistance to trastuzumab remain sensitive to trastuzumab anti-tumor effects in vivo and to ADCC killing. Cancer Immunol Immunother 58: 1887-1896, 2009.

42. Arnould L, Gelly M, Penault-Llorca F, et al: Trastuzumab-based treatment of HER 2-positive breast cancer: An antibody-dependent cellular cytotoxicity mechanism? Br J Cancer 94: 259-267, 2006.

43. Varchetta S, Gibelli N, Oliviero B, et al: Elements related to heterogeneity of antibody-dependent cell cytotoxicity in patients under trastuzumab therapy for primary operable breast cancer overexpressing Her2. Cancer Res 67: 11991-11999, 2007.

44. Akiyama S, Furukawa T, Sumizawa T, et al: The role of thymidine phosphorylase, an angiogenic enzyme, in tumor progression. Cancer Sci 95: 851-857, 2004.

45. Albanell J, Codony J, Rovira A, Mellado B and Gascón P. Mechanism of action of anti-HER2 monoclonal antibodies: Scientific update on trastuzumab and 2C4. Adv Exp Med Biol 532: 253-268, 2003. 